\title{
CLASS GROUPS OF RANK ONE SEMISIMPLE MONOIDS
}

\author{
LEX E. RENNER
}

(Communicated by Louis J. Ratliff, Jr.)

\begin{abstract}
Let $M$ be an irreducible, normal, algebraic monoid with unit group $S l_{2}(\mathscr{l}) \times \mathscr{\ell}^{*}, G l_{2}(\mathscr{\ell})$ or $P G l_{2}(\not{k}) \times \ell^{*}$. In [7] these monoids are classified numerically. In this paper we compute explicitly the divisor class group of each monoid. As a corollary we characterize the monoids with factorial coordinate algebra. All results are independent of the characteristic of $h$.
\end{abstract}

Introduction. In [7] the classification problem for semisimple rank one algebraic monoids is solved numerically. Associated with each semisimple monoid $M$, with unit group $G=G l_{2}(\not), S l_{2}(\not) \times k^{*}$ or $P G l_{2}(k) \times k^{*}$, is its polyhedral root system $(X, \Phi, C)$, which is a complete and discriminating invariant of $M$. Furthermore, for each $G$ there is a canonical one-to-one correspondence between the essentially distinct polyhedral root systems and the set of positive rational numbers [7]. Thus, each $M$ is isomorphic to a unique $M_{r}, r \in \mathbf{Q}^{+}$.

The purpose of this paper is to interpret the correspondence $M \mapsto r, r \in \mathbf{Q}^{+}$, in terms of more conventional invariants of $M$; in particular, the divisor class group $[2]$.

To state the main result let $M=M_{r}, r \in \mathbf{Q}^{+}$, be a semisimple monoid with $G$ as above, and write $r=s / t$ where $(s, t)=1$. Let $\mathrm{Cl}(M)$ be the divisor class group of $M$, and let $\mathbf{Z}_{s}$ denote the integers modulo $s$.

THEOREM. (a) If $G=S l_{2}(\not) \times k^{*}$ then $\mathrm{Cl}(M) \cong \mathbf{Z}_{s}$.

(b) If $G=G l_{2}(\not k)$ then

$$
\mathrm{Cl}(M) \cong \begin{cases}\mathbf{Z}_{2 s} & \text { if } 2 \mid s t \\ \mathbf{Z}_{s} & \text { if } 2 \nmid s t\end{cases}
$$

(c) If $G=P G l_{2}(\not k) \times k^{*}$ then $\mathrm{Cl}(M) \cong \mathbf{Z}_{s} \oplus \mathbf{Z}_{2}$.

One major technique in the proof is the big cell $U \subseteq M$, which was constructed in [7] for a different purpose. In this paper, it is used to reduce our problems to numerical questions about the polyhedral root system.

Background. An algebraic monoid is an affine algebraic variety $M$ defined over the algebraically closed field $k$, together with an associative morphism $\mu: M \times M \rightarrow$ $M$ and a two-sided unit $1 \in M$ for $\mu$. We assume $M$ is irreducible as a variety. In this case, $G=\left\{x \in G \mid x^{-1} \in M\right\} \subseteq M$ is open and dense in the Zariski topology [4]. $E(M)=\left\{e \in M \mid e^{2}=e\right\} \subseteq M$ is the set of idempotents of $M$. A submonoid $Z \subseteq M$ is a $D$-submonoid if $Z=\bar{T}$, the Zariski closure of some torus $T \subseteq G$. By

Received by the editors February 2, 1987 and, in revised form, March 27, 1987.

1980 Mathematics Subject Classification (1985 Revision). Primary 20M99, 14M20.

Supported by the NSERC. 
[5, Corollary 2], every idempotent $e$ of $M$ is an element of some $D$-submonoid $Z$ of $M$. Note that every $D$-monoid $Z$ is uniquely determined by its character monoid $X(Z)=\{\chi \in R[Z] \mid \chi: Z \rightarrow h$ is a morphism of monoids $\}$. An irreducible monoid $M$ is semisimple if $0 \in M, M$ is normal, and $\operatorname{dim} Z G=1$, where $Z G$ is the center of $G$ [7]. In this paper, we are concerned mainly with semisimple monoids with unit group $G l_{2}(k), S l_{2}(k) \times k^{*}$ or $P G l_{2}(k) \times k^{*}$. Such monoids will be called semisimple rank one.

Main results. If $R$ is a Noetherian domain, integrally closed in its fraction field, we define the divisor class group, $\mathrm{Cl}(R)$ (as in [2]) to be the group of divisorial ideals of $R$ modulo the group of principal ideals. If $R=k[X]$ for some normal affine variety $X$, we put $\mathrm{Cl}(X)=\mathrm{Cl}(R)$. Recall that any pure codimension one subvariety $D$ of $M$ determines an element $D \in \mathrm{Cl}(X)$. [2].

3.1 LeMMA. Let $M$ be a semisimple rank one monoid and let $D \in \mathrm{Cl}(M)$ be the divisor class of $M \backslash G \subseteq M$. Then

(a) $0 \rightarrow \mathbf{Z} \cdot D \rightarrow \mathrm{Cl}(M) \rightarrow \mathrm{Cl}(G) \rightarrow 0$ is exact.

(b) $\mathrm{Cl}(M)$ is finite.

ProOF. (a) follows from Nagata's theorem [2], since by [7, Corollary 3.3], $M \backslash G$ is irreducible. Now by [6, Theorem 3.4], there exists $\chi: M \rightarrow k$ such that $\chi^{-1}(0)=M \backslash G$. Thus, in $\operatorname{Cl}(M)$, some multiple of $D$ is zero, so that $\mathbf{Z} \cdot D$ is finite. Hence, using (a), $\mathrm{Cl}(M)$ is finite, since by $[\mathbf{3}], \mathrm{Cl}(G) \cong \mathrm{Z}_{2}$ if $G=P G l_{2}(\not k) \times \ell^{*}$ and $(0)$ otherwise.

3.2 LEMMA. Let $M$ be as in 3.1 and let $X(M)=\{\chi: M \rightarrow k \mid \chi$ is a morphism of algebraic monoids $\}$. So $j: X(M) \subseteq X(G)$ by restriction. Then $X(M) \cong \mathbf{N}$ and $X(G) \cong \mathbf{Z}$ in such a way that $j$ is the usual inclusion.

Proof. By [6], $X(M) \neq \varnothing$ so let $X^{\prime}(M)=\left\{\chi \in X(G) \mid \chi^{n} \in X(M)\right.$ some $\left.n\right\}$. But then $X(M)=X^{\prime}(M)$ since $M$ is normal. It follows that $X(M), X(G)$ and $j$ are as stated.

Let $\bar{T} \subseteq M$ be the Zariski closure in $M$ of a maximal torus $T$ of $G$. By [8] there exist unique $V, Y \in X(\bar{T})$ such that

(a) For all $\chi \in X(\bar{T})$ there exists $k, l, m \in \mathbf{N}$ such that $\chi^{m}=V^{k} Y^{l}$.

(b) If $\{g, h\} \subseteq X(\bar{T})$ satisfies (a) then $g=V^{s} Y^{t}$ and $h=V^{u} Y^{v}$ for appropriate $s, t, u, v, \in \mathbf{N}$.

$\{V, Y\}$ is the set of fundamental generators.

Let $\bar{T}=T \cup T e \cup T f \cup\{0\}$, as in $[7, \S 3]$. Then $\not[\bar{T}]\left[V^{-1}\right]$ is the coordinate algebra of the open submonoid $T_{e}=T \cup T e$, and similarly for $k[\bar{T}]\left[Y^{-1}\right]$ and $T_{f}=T \cup T f$. By [1, Comment 3.3], or a direct argument, $T_{e} \cong \not k \times k^{*}$ (and similarly for $T_{f}$ ). In particular, $k\left[T_{e}\right]$ and $k\left[T_{f}\right]$ are UFDs, and so

(i) $\left\{\Psi \in \mathscr{k}\left[T_{e}\right]|\Psi|_{T_{e} \backslash T}=0\right\}$ is a principal ideal of $\mathscr{k}\left[T_{e}\right]$, and

(ii) $X\left(T_{e}\right) \cong \mathbf{Z} \oplus \mathbf{N}$.

3.3 MAIN LEMMA. Let $\bar{T}$ be as above, and let $\chi \in X\left(T_{e}\right)$ be the restriction to $T_{e}$ of the generator $g \in X(M) \cong \mathbf{N}$ (as in 3.2). Write $\chi=U^{s} V^{m} \in X\left(T_{e}\right)=$ $\left\{U^{a} V^{b} \mid a \in \mathbf{N}, b \in \mathbf{Z}\right\}$. Then the order of $D \in \mathrm{Cl}(M)$ (as in 3.1) is s.

Proof. Let $B=\{g \in G \mid g e=e g e\} \subseteq G$. Then by [7, Proposition 2.4], $B$ is a Borel subgroup and $B^{-}=\{g \in G \mid e g=e g e\}$ is opposite to $B$ relative to $T$. 
Furthermore, by [7, Proposition 3.2],

$$
\tau: U^{-} \times T_{e} \times U \rightarrow M, \quad \tau(u, y, v)=u y v
$$

is an open embedding, where $U$ and $U^{-}$are the unipotent radicals of $B$ and $B^{-}$respectively. Similarly $\nu: U \times T_{f} \times U^{-} \rightarrow M, \nu(u, y, v)=u y v$, is an open embedding. Let $\mathscr{V}_{e}=\operatorname{image}(\tau)$ and $\mathscr{V}_{f}=\operatorname{image}(\nu)$. Note that $\mathscr{V}_{e}$ and $\mathscr{V}_{f}$ are both affine open subsets of $M$, while $\mathrm{Cl}(M)$ is finite. It follows that there exist $r, s \in \not{k}[M]$ such that $k\left[\mathscr{V}_{e}\right]=k[M][1 / r]$ and $k\left[\mathscr{V}_{f}\right]=k[M][1 / s]$. Furthermore, height $(r, s)=2$, since by [7, §2], $M \backslash \mathscr{V}_{e}$ and $M \backslash \mathscr{V}_{f}$ are both irreducible closed subsets of $M$. Now let

$$
\mu=\left\{g \in \mathscr{R}[M]|g|_{M \backslash G}=0\right\} .
$$

Then

$$
o(D)=\inf \left\{s \in \mathbf{N} \mid \mu^{(s)} \text { is principal }\right\}
$$

where $\mu^{(s)}$ denotes the sth symbolic power of $\mu$ [2]. On the other hand, if $\mu^{(s)}$ is principal, then $\mu^{(s)}=(\chi)$ for some $\chi \in X(M)$, since these are the only principal ideals of $k[M]$ stable under both right and left translation by $G$. Thus, if $\chi \in$ $X(M) \cong \mathbf{N}$ is the generator, then $o(D)=s$, where $(\chi)=\mu^{(s)}$. Finally, $\mathscr{V}_{e} \rightarrow$ $M$ induces $\left.k[M] \rightarrow k\left[\mathscr{V}_{e}\right]=k[M][1 / r] \cong \not A^{-}, U, V, V^{-1}, A\right]$, where the last. isomorphism results from the fact that $\mathscr{V}_{e} \cong \not k \times\left(\not{k} \times \mathscr{k}^{*}\right) \times k$. Now $\mu \cdot \boldsymbol{k}\left[\mathscr{V}_{e}\right]=(u)$ for some prime element $u \in k[M][1 / r]$, since $k\left[\mathscr{V}_{e}\right]$ is a UFD. Thus, $\left.(\chi) \not \mathscr{V}_{e}\right]=$ $\left.\mu^{(s)} \not \mathscr{V}_{e}\right]=(u)^{(s)}=\left(u^{s}\right)$. Hence, $\chi=u^{s} \varsigma$ for some unit $\varsigma \in \mathscr{k}\left[\mathscr{V}_{e}\right]$. But then $\varsigma=V^{m}$, some $m$ and thus, $\chi=U^{s} V^{m} \in \mathcal{k}\left[T_{e}\right]$.

3.4 COROLlaRY. Let $\mu=\left\{\Psi \in k[\bar{T}]|\Psi|_{\bar{T} \backslash T}=0\right\}$. Then $\mu$ is principal iff $\rho$ is principal iff $\mathrm{Cl}(M)=\mathrm{Cl}(G)$.

ProOF. $\mu$ is principal iff $\chi=U V^{m} \in \not\left[T_{e}\right]=\not\left[U, V, V^{-1}\right]$ iff $\chi=X Y^{m} \in$ $\left.\not T_{f}\right]=\not{k}\left[X, Y, Y^{-1}\right]$. So $\mu$ is principal if and only if $\mu k\left[T_{e}\right]$ and $\rho \not k\left[T_{f}\right]$ are principal ideals generated by $\chi$. But height $(V, Y)=2$ as an ideal of $\not k[\bar{T}]$. Thus, $(\chi)=\mu$.

3.5. Let $M$ be a semisimple rank one monoid with unit group $G$ and maximal torus $T \subseteq G$, and let $\bar{T} \subseteq M$. Then by [7, Theorem 5.3], $M=M_{r}$, for some $r \in \mathbf{Q}^{+}$ and by $[\mathbf{7}, \S 4.5]$,

(a) $X(\bar{T}) \cong X(r)=\{(b, a) \in X(T)=\mathbf{Z} \oplus \mathbf{Z}|| a / b \mid \leq r\} \cup\{(0,0)\}$ if $G=$ $S l_{2}(k) \times k^{*}$.

(b) $X(\bar{T}) \cong Y(r)=\{(b, a) \in X(T)=\mathbf{Z} \oplus \mathbf{Z}||(a-b) /(a+b) \mid \leq r\} \cup\{(0,0)\}$ if $G=G l_{2}(k)$.

(c) $X(\bar{T})=X(r)$ if $G=P G l_{2}(\not) \times k^{*}$.

Note. The "axes" have been interchanged in this paper. In cases (a) and (c) $\chi=(1,0) \in X(\bar{T})$ is the character that extends to the generator of $X(M)=\mathbf{N}$ (as in 3.2$)$. In case (b), $\chi=(1,1)$.

3.6 LEMMA. (a) Suppose $m, n, a, b \in \mathbf{Z}$ and $m b-n a=1$. If $\alpha(n, m)+\beta(b, a)=$ $(1,0)$ then $\beta=m$.

(b) Suppose instead that $\alpha(n, m)+\beta(b, a)=(1,1)$. Then $\beta=m-n$.

ProOF. Obvious. 
3.7 THEOREM. Let $M=M_{r}, r \in \mathbf{Q}^{+}$, be a semisimple, rank one, algebraic monoid, and let $D \in \mathrm{Cl}(M)$ be as in 3.1. Write $r=s / t$ where $(s, t)=1$. Then $o(D)=s$, unless $G=G l_{2}(\not k)$ and $2 \nmid s t$, in which case $o(D)=2 s$.

PROOF. Let $V, Y \in X(\bar{T})$ be the fundamental generators, and assume that $G=G l_{2}(k)$ (the other cases are slightly easier). By $3.5(\mathrm{~b}), V=(n, m)$, where $(m-n) /(m+n)=r$. Let $(b, a) \in X(\bar{T})$ such that $m b-n a=1$. By 3.6(b), if $\alpha(n, m)+\beta(b, a)=(1,1)$ then $\beta=m-n$, and so by $3.3, o(D)=m-n$. Now if $m$ and $n$ are not both odd, then $(m-n, m+n)=1$, and so $m-n=s$ and $m+n=t$. If $m$ and $n$ are both odd then $(m-n, m+n)=2$ and so $(m-n) / 2=s$ and $(m+n) / 2=t$. So in this case, $m-n=2 s$ and $m+n=2 t$.

3.8 THEOREM. Let $M=M_{r}$ be a semisimple rank one monoid with unit group G. Write $r=s / t,(s, t)=1$.

(a) If $G=S l_{2}(\not k) \times \not^{*}$ then $\mathrm{Cl}(M) \cong \mathbf{Z}_{s}$.

(b) If $G=G l_{2}(k)$ then

$$
\mathrm{Cl}(M) \cong \begin{cases}\mathbf{Z}_{2 s} & \text { if } 2 \mid s t, \\ \mathbf{Z}_{s} & \text { if } 2 \nmid s t .\end{cases}
$$

(c) If $G=P G l_{2}(\not k) \times \mathscr{k}^{*}$ then $\mathrm{Cl}(M) \cong \mathbf{Z}_{s} \oplus \mathbf{Z}_{2}$.

Proof. Cases (a) and (b) follow from 3.1 and 3.7 since $\mathrm{Cl}(G)=(0)$ in these cases [3]. For (c) we distinguish two cases. If st is odd then by 3.1 and $3.7, \mathrm{Cl}(M)$ is an extension of a cyclic group of odd order by $\mathbf{Z}_{2}$. But any such extension splits. If $s t$ is even consider the map $\Psi: G l_{2}(\not k) \rightarrow P G l_{2}(\not k) \times \not^{*}$ of degree two (factor out the central subgroup of $G l_{2}(\not k)$ of order two). Let $\Psi: M^{\prime} \rightarrow M$ be the normalization of $M$ along $\Psi$ (see [8, Lemma 7.1.1]). It follows from 3.5(b) that $M^{\prime}$ also corresponds to $r$ for the group $G l_{2}(\ell)$. It is also easy to check that $\Psi^{*}: X(M) \rightarrow X\left(M^{\prime}\right)$ is bijective. Thus, by $3.3 \mathrm{Cl}(\Psi): \mathbf{Z} \cdot D \rightarrow \mathbf{Z} \cdot D^{\prime}$ is injective and by 3.7 it is surjective since these groups have the same order. Thus we have the desired splitting

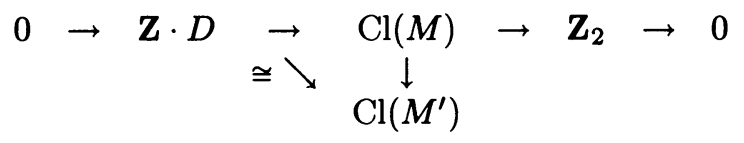

This concludes the proof.

3.9 COROLLARY. Let $M=M_{r}$ be a semisimple rank one monoid with unit group $G$. Then the following are equivalent.

(a) $\mathscr{k}[M]$ is factorial.

(b) $G=S l_{2}(k) \times \ell^{*}$ and $r=1 / t$, or $G=G l_{2}(k)$ and $r=1 / t$ with $2 \nmid t$.

\section{REFERENCES}

1. V. I. Danilov, The geometry of toric varieties, Russian Math. Surveys 33:2 (1978), 97-154.

2. R. Fossum, The divisor class group of a Krull domain, Ergebnisse Math. Grenzgeb., Band 74, Springer-Verlag, New York, 1973.

3. B. Iverson, The geometry of algebraic groups, Advances in Math. 20 (1976), 57-85.

4. M. Putcha, On linear algebraic semigroups, Trans. Amer. Math. Soc. 259 (1980), 457-469.

5. _ Green's relations on a connected algebraic monoid, Linear and Multilinear Algebra 11 (1982), 205-214. 
6. L. Renner, Quasi-affine algebraic monoids, Semigroup Forum 30 (1984), 167-176.

7. Classification of semisimple rank one monoids, Trans. Amer. Math. Soc. 287 (1985), 457-473.

8. _ Classification of semisimple algebraic monoids, Trans. Amer. Math. Soc. 292 (1985), 193-223.

DEPARTMENT OF MATHEMATICS, UNIVERSITY OF WESTERN ONTARIO, LONDON, ONTARIO N6A 5B7, CANADA 